\title{
Epidemiology of dermatomycoses in southwest Poland, years 2011-2016
}

\author{
Anna Gawdzik, Katarzyna Nowogrodzka, Anita Hryncewicz-Gwóźdź, Joanna Maj, Jacek Szepietowski, \\ Alina Jankowska-Konsur
}

Department of Dermatology, Venereology and Allergology, Wroclaw Medical University, Wroclaw, Poland

Adv Dermatol Allergol 2019; XXXVI (5): 604-608

DOI: https://doi.org/10.5114/ada.2018.80615

\begin{abstract}
Introduction: Superficial mycosis is one of the most common diseases worldwide, however its epidemiology is changing over time.

Aim: To present epidemiological data of the skin fungal infections diagnosed in the years 2011-2016 in Lower Silesia. Material and methods: A total of 11004 patients with a clinically suspected superficial mycosis were investigated. Skin scrapings, nail clippings and plucked hair were examined with a direct microscopy, Wood's lamp and culture. Particular species were identified via polymerase chain reaction (PCR) examination. The lesions suspected for pityriasis versicolor were screened for Malassezia with Wood's lamp and direct microscopy.

Results: Dermatomycosis was diagnosed in 1653 (15.00\%) patients with 1795 fungi identified. 1858 specimens were indicative of fungal infection including dermatophytes, yeasts and moulds. Out of 924 cases of dermatophytic infections (51.48\%), Trichophyton rubrum accounted for the majority (71.75\%) and was followed by Trichophyton tonsurans (16.77\%). Among the yeasts (716; 39.89\%), Candida spp. was the most common agent identified (521; $67.66 \%)$. The sites affected most often were toenails $(956 ; 51.45 \%)$ and fingernails $(319 ; 17.17 \%)$. In paediatric population the most common diagnosis was tinea corporis (60, 41.10\%).

Conclusions: Our study revealed that toenail onychomycosis remains the most common superficial mycosis and T. rubrum is the most common pathogen. However, in a longer period of observation, a decrease in the number of tinea capitis cases and an increase in infections caused by T. tonsurans were noticed. Observed changes indicate the need for continuing studies to detect the upcoming epidemiological trends.
\end{abstract}

Key words: dermatomycoses, dermatophytes, yeasts, moulds, Poland, epidemiology.

\section{Introduction}

Superficial mycosis is reported to be one of the most prevalent diseases with $20-25 \%$ of the world's population affected [1]. It is caused by a heterogeneous group of pathogens including dermatophytes, yeasts and moulds; the distribution of the pathogens changes with time due to increased migration and lifestyle changes as well as varies between continents, climate zone, age groups and sites affected. Improved socioeconomic conditions, population ageing and medical care with an access to antimicrobial, antifungal and immunosuppressive drugs have contributed to a slow but inevitable shift in epidemiology.

\section{Aim}

Herein, we present epidemiological data of the skin fungal infections diagnosed over the past 5 years in the
Mycological Laboratory of the Wroclaw Medical University Hospital, Lower Silesia and we compare our findings with those previously reported from the same region.

\section{Material and methods}

In the years 2011-2016, 11004 patients were examined in the Mycological Laboratory of Dermatology, Venereology and Allergology Department of the Wroclaw Medical University. Skin scales, hair and nail clippings were collected from the sites clinically suspected of fungal infection. Subsequently, the material was exposed to a solution of $20 \%$ potassium hydroxide and dimethyl sulfoxide (DMSO) to provide a visualization of fungal elements with a direct microscopy. Then, the cultures of dermatophytes were performed on Sabouraud agar and incubated for 4 weeks at room temperature.

\footnotetext{
Address for correspondence: Alina Jankowska-Konsur MD, PhD, Department of Dermatology, Venereology and Allergology, Wroclaw Medical University, 1 Chałubińskiego St, 50-368 Wroclaw, Poland, phone: +48 7178422 86,

e-mail: alina.jankowska-konsur@umed.wroc.pl

Received: 9.07.2018, accepted: 7.08.2018.
} 
Further identification was based on a macroscopic and microscopic image of the colonies. In selected cases polymerase chain reaction (PCR) was performed. Lesions suspected of pityriasis versicolor were examined in a direct microscopy and a Wood's light fluorescence. Seborrheic dermatitis was excluded from the survey. Some of the patients had a fungal co-infection or presented with more than one body area affected.

\section{Statistical analysis}

Statistical analysis was performed with the $\chi^{2}$ test. $P$-values of less than 0.05 were considered statistically significant. Two tables and a diagram were added for more transparency.

\section{Results}

Out of the 11004 individuals examined, 1653 (15.00\%) patients, 798 (48.30\%) men and 855 (52.10\%) women were affected by a superficial fungal infection. The age range was from 6 months to 96 years with the mean age of 47 years. A group of 139 (8.41\%) patients were under 18 years of age. 641 (38.80\%) patients were residents of a city with a population of at least 100000 , 412 (24.90\%) lived in smaller cities and 327 (19.80\%) of them lived in rural areas. For the remaining 273 cases, the data were not known. A total of 1858 specimens were collected. In a diagnostic process 1795 strains were identified. Two hundred five patients (12.4\%) had more than one site affected. A total of 63 (3.79\%) patients had the same strain identified in more than one localization. A fungal co-infection was revealed in 136 (7.57\%) cases. A correspondence of $89.03 \%$ between a positive direct mycological examination and a culture was obtained.

Dermatophytes accounted for $51.48 \%(n=924)$ of strains and were represented mostly by anthropophilic species ( $n=830 ; 89.83 \%)$. Zoophilic $(n=91 ; 9.85 \%)$ and geophilic species $(n=3 ; 0.32 \%)$ were less frequent. Yeastlike fungi infections were less common ( $n=716$; 39.89\%). Candida species, including C. albicans, C. tropicalis and C. krusei, were isolated most frequently ( $n=521 ; 72.77 \%)$.

Mould strains present in $8.64 \%(n=155)$ of cases were regarded as a contamination of a specimen. The exact data are given in Table 1.

Out of 1858 specimens collected from different body areas, onychomycosis was the most common diagnosis with the highest prevalence in patients aged 50 to 64 years (Figure 1). Toenails were affected in 956 (51.45\%) cases and fingernails in 319 (17.17\%) cases. Toenail onychomycosis was statistically more common in the male population $(p=0.0007)$. The opposite relation was noticed as far as fingernails were concerned. In regard to toenails, dermatophytes were diagnosed in $69.35 \%$ of specimens ( $n=663)$, mostly T. rubrum $(n=535,55.96 \%)$, followed by $T$. tonsurans ( $n=94 ; 9.83 \%$ ) (Table 2$)$. The majority of yeast-like toenail infections ( $n=167 ; 17.47 \%$ ) were due to Candida sp. ( $n=141 ; 14.75 \%)$. Contrarily, yeasts were a more common cause of fingernail onychomycosis than dermatophytes $(n=251 ; 78.86 \%$ vs. $n=53$; $16.61 \%)$. Candida sp. $(n=241 ; 75.55 \%)$ was the main pathogen isolated from this site. T. rubrum was detected in $13.48 \%$ of specimens $(n=43)$.

The glabrous skin was the third most frequent site of infection ( $n=296 ; 15.93 \%$ ). The most affected age group was young adults, 18 to 34 years old $(n=103$, $34.8 \%$ ). $55.51 \%$ ( $n=163$ ) of specimens were positive for yeasts with the highest prevalence of Malassezia sp. $(n=152 ; 51.35 \%)$. Dermatophytes, found in 129 specimens (43.58\%) were statistically less frequent ( $p=0.046)$. The majority of infections were caused by T. rubrum ( $n=59 ; 19.93 \%$ of dermatophytes). In paediatric population it was the first most common site of infection ( $n=60 ; 41.10 \%)$.

Twenty-five (1.35\%) patients were diagnosed with tinea capitis. Eighty-four percent of them were younger than 18 years of age with the highest prevalence in children aged from 6 to 11 years $(n=14,56.76 \%)$. Females were more frequently affected, with a female to male ratio of $3: 2$. Dermatophytic infections were the most common ( $n=19,76.00 \%$ ) with the highest prevalence of zoophilic fungi, mainly $M$. canis ( $n=8,32.00 \%)$. Anthropophilic dermatophytes, mainly $T$. tonsurans, were detected in $24.00 \%(n=6)$ of cases.

Fourty $(2.15 \%)$ patients diagnosed with tinea faciei were mostly from the paediatric population $(n=25$; $62.50 \%$ ), especially from the 6 - to 11 -year-old group $(n=16 ; 40.00 \%)$. Dermatophytes were found in the majority of specimens ( $n=35$; $87.50 \%$ ) with a zoophilic fungi Trichophyton mentagrophytes (var. granulosum and var. mentagrophytes) as the primary causative agent ( $n=14 ; 35.00 \%)$, followed by anthropophilic dermatophyte T. tonsurans ( $n=11 ; 24.00 \%)$. Yeasts were diagnosed in $7.50 \%$ of cases $(n=3)$.

A total number of 67 (3.49\%) specimens positive for tinea pedis and 12 cases of tinea manuum (0.63\%) were diagnosed with a majority of patients from the adult population (about $91.36 \%$ for both locations). Tinea manuum was more common in the female population $(n=8$, $66.67 \%$ ), yet the group was rather small so the result may not be representative. No such predilection was noticed for tinea pedis. T. rubrum was the most prevalent dermatophyte in both feet $(n=36 ; 53.73 \%)$ and hands ( $n=3,25.00 \%$ ). $41.67 \%$ of patients with tinea manuum were positive for Candida sp. $(n=3 ; 25.00 \%)$.

In the group of $32(1.72 \%)$ cases of tinea cruris, the majority of patients (39.02\%) were adults $(n=30$; 93.75\%) mostly from the 50-64-year-old group (40.63\%) and only 2 patients were younger than 18 years old (6.25\%). The male to female ratio was 9.6 : 1 . Dermatophytes were diagnosed more often than yeasts $(n=20$; $62.50 \%$ vs. $n=11 ; 34.38 \%$ ). Of all pathogens T. rubrum was the most prevalent one; it was detected in $56.25 \%$ of 
Table 1. Fungal species diagnosed, years 2011-2016

\begin{tabular}{|c|c|c|c|}
\hline Fungal species & Number of pathogens & $\begin{array}{l}\text { Percentage of groups } \\
\text { (\%) }\end{array}$ & $\begin{array}{l}\text { Percentage of all } \\
\text { pathogens (\%) }\end{array}$ \\
\hline Zoophilic: & 91 & 9.85 & \\
\hline Trichophyton mentagrophytes var. granulosum & 32 & 3.46 & 1.73 \\
\hline $\begin{array}{l}\text { Trichophyton mentagrophytes var. } \\
\text { mentagrophytes }\end{array}$ & 34 & 3.68 & 1.84 \\
\hline Microsporum canis & 25 & 2.71 & 1.35 \\
\hline Anthropophilic: & 830 & 89.83 & \\
\hline Trichophyton rubrum & 663 & 71.75 & 35.88 \\
\hline Trichophyton tonsurans & 155 & 16.77 & 8.39 \\
\hline Epidermophyton floccosum & 11 & 1.19 & 0.60 \\
\hline Trichophyton cutaneum & 1 & 0.11 & 0.05 \\
\hline Geophilic: & 3 & 0.32 & \\
\hline Microsporum gypseum & 3 & 0.32 & 0.16 \\
\hline All dermatophytes & 924 & 100 & 51.48 \\
\hline Candida sp. & 521 & 72.77 & 29.03 \\
\hline Rhodotorula rubra & 34 & 4.75 & 1.89 \\
\hline Geotrichum sp. & 7 & 0.89 & 0.39 \\
\hline Trichosporon sp. & 1 & 0.14 & 0.06 \\
\hline Malassezia sp. & 153 & 21.37 & 8.52 \\
\hline All yeasts & 716 & 100 & 39.89 \\
\hline All moulds & 155 & 100 & 8.64 \\
\hline All groups & 1795 & & 100 \\
\hline
\end{tabular}

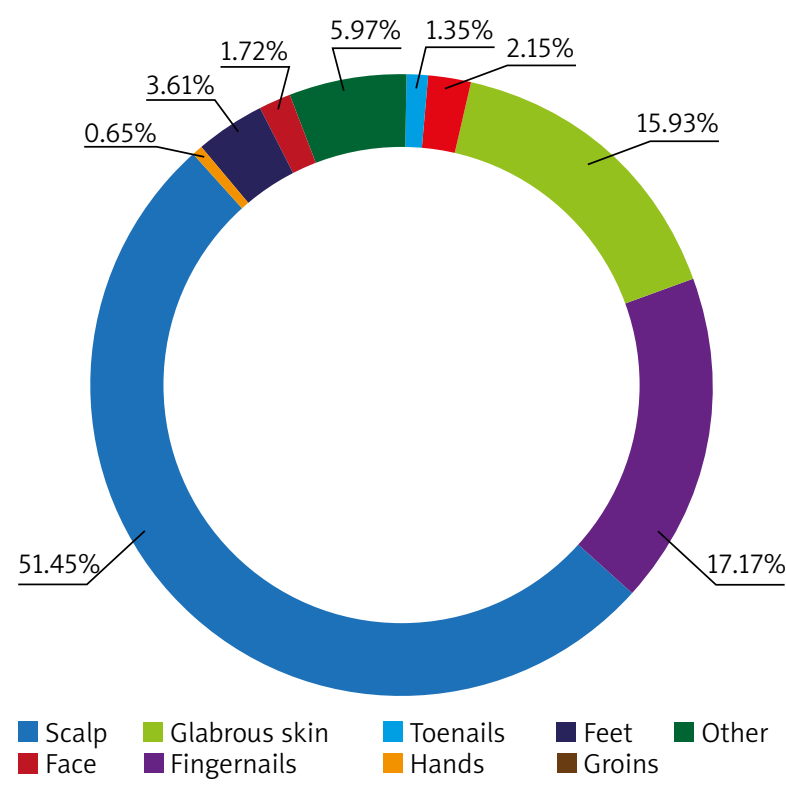

Figure 1. Distribution of superficial fungal infections cases ( $n=18)$ while Candida sp. was found in $11(34.38 \%)$ specimens.

$5.59 \%(n=114)$ of infections involved other locations, mainly the genital area and oral cavity mucosa. $93.37 \%$ of specimens were positive for yeasts, primarily Candida sp. $(n=108 ; 94.74 \%)$. This localization was uncommon in children with only 2 patients from the paediatric population (1.75\%).

\section{Discussion}

A vast range of dermatomycoses strikes the importance of monitoring of the ongoing changes in the epidemiology. We will summarize briefly the most important outcomes of the analysis of the present and previously conducted surveys in the southwest Poland and compare them with the global epidemiological situation.

In years 2011-2016, the rate of superficial fungal infections diagnosed in the Dermatology Department of the Wroclaw Medical University was $15.00 \%$ and as compared to a survey conducted in the Lower Silesia region 
in years 2003-2007, it decreased from 23.5\% [2] and yet, the difference was not statistically significant $(p=0.417)$. Toenail onychomycosis remained the most frequent diagnosis and there was no statistical difference in its prevalence between 2003-2007 and 2011-2016 ( $p=0.231$ ). An anthropophilic dermatophyte, T. rubrum, was the major etiological agent of toenail onychomycosis also in other countries such as Germany [3] and the United Kingdom [4]. In the latter study, fingernail onychomycosis was in most cases due to T. rubrum [4] while our study revealed Candida sp. as the main causative agent. According to the literature, toenails were affected ten times more often [5], whereas in our survey the proportion was $3: 1$. Most studies suggested gender predilection towards males in toenail onychomycosis [5] and female predilection as far as fingernails were concerned $[3,4]$. The same results were evident in our survey.

Zoophilic dermatophyte, $M$. canis, is considered the major causative agent of tinea capitis worldwide and in Europe [6], yet the epidemiological situation varies between countries. It remains the most common cause of scalp ringworm in Poland [7, 8], Germany [2], southern Europe, including Spain [8] and Greece [9]. Dermatophytes of anthropophilic transmission are geographically restricted [6], for instance, T. tonsurans is common in Canada, the USA [1] and in Latin American countries [10], while T. violaceum is the most prevalent pathogen in Africa. M. audouinii and T. soudanense are also common in Africa [3]. In regard to the present epidemiological situation in Lower Silesia, in comparison with data from past decades obtained in surveys conducted in the Department of Dermatology, Venereology and Allergology of Wroclaw Medical University in years 1974-1991 [11] and 2002-2006 [7], a decrease in the incidence of dermatophyte infections of the scalp was noted $(p=0.033)$. However, there was no statistical difference between the present results and the situation reported in the previous study based in Lower Silesia [7] ( $p=0.236)$. M. canis is still the most prevalent causative agent $[7,11]$. Since 1974 the prevalence of T. tonsurans in southwest Poland had been gradually increasing from $3.1 \%$ in the years $1974-1991$ to $31.58 \%$ in the years $2011-2016$ ( $p=0.0006$ ), being now the second most common cause of scalp ringworm in Lower Silesia. This trend is also visible in the urban areas of France [12] and the United Kingdom [13, 14], where it has become the main cause of scalp ringworm. The pattern is changing mainly due to modern lifestyle and hygiene improvement [1]. In some countries, for instance in Spain [8], the second causative agent remains T. mentagrophytes, just as it used to be reported in Poland in the past decades [11]. Due to migration from the northern Africa, an increase in T. violaceum prevalence was noted in some European countries, like Spain [6, 15], Italy [16] and Switzerland [17]. For the same reason, the prevalence of $M$. audouinii was rising in France and in the UK [12]. The trend had no influence on epidemiology of

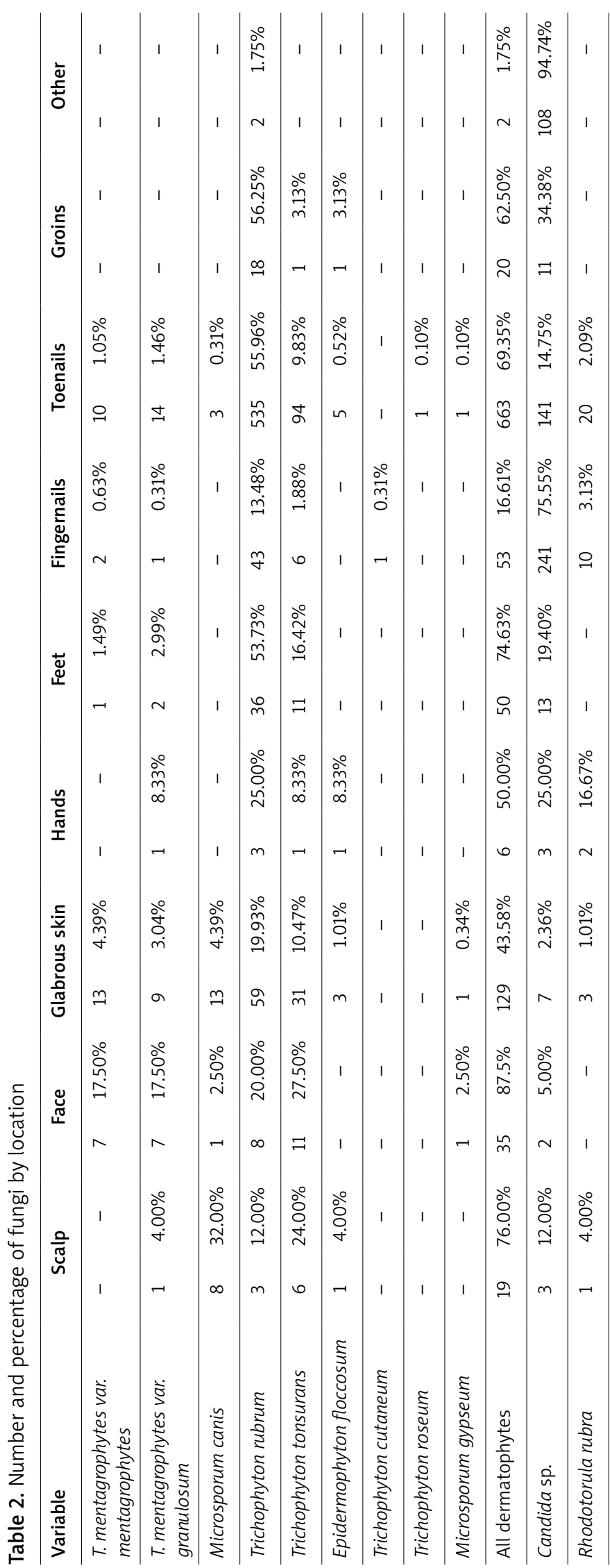


dermatomycoses in Lower Silesia, Poland, where infections caused by T. violaceum and $M$. audouinii had been rare in past 40 years [7] and in the present investigation no new cases were reported. Moreover, there had been no evidence of T. soudanense in the population of southwest Poland either in the recent study or in the past [7].

As might be expected, there are certain differences in epidemiology of tinea corporis, sometimes even between bordering countries or within one country. While in Lower Silesia, T. rubrum was the most prevalent agent, in Lithuania [18] most cases were due to $M$. canis. In Teheran, Iran, T. tonsurans was most often reported, however in the northeast of Iran T. mentagrophytes was the main etiological agent [19].

\section{Conclusions}

Our study revealed that toenail onychomycosis remains the most common superficial mycosis, as well as T. rubrum is the most common pathogen. However, in a longer period of observation, a decrease in number of tinea capitis cases and an increase in infections caused by $T$. tonsurans were noticed. Observed changes indicate the need for continuing studies to detect the upcoming epidemiological trends.

\section{Acknowledgments}

Anna Gawdzik and Katarzyna Nowogrodzka equally contributed to the work.

\section{Conflict of interest}

The authors declare no conflict of interest.

\section{References}

1. Zhan P, Liu W. The changing face of dermatophytic infections worldwide. Mycopathologia 2017; 182: 77-86.

2. Jankowska-Konsur A, Dyląg M, Hryncewicz-Gwóźdź A, et al. A 5-year survey of dermatomycoses in southwest Poland, years 2003-2007. Mycoses 2009; 54: 162-7.

3. Nenoff P, Krüger C, Ginter-Hanselmayer G, et al. Mycology - an update. Part 1: dermatomycoses: cusative agents, epidemiology and pathogenesis. J Dtsch Dermatol Ges 2014; 12: 188-210.

4. Wlodek C, Trickey A, De Berker D, et al. Trends in laboratorydiagnosed onychomycosis between 2006 and 2014 in the South West of England. BJD 2017; 176: 237-40.

5. Faergemann J, Baran R. Epidemiology, clinical presentation and diagnosis of onychomycosis. BJD 2003; 149 (Suppl 65): 1-4.

6. Ginter-Hanselmayer G, Weger W, Ilkit M, et al. Epidemiology of tinea capitis in Europe: current state and changing patterns. Mycoses 2007; 50: 6-13.

7. Jankowska-Konsur A, Dyląg M, Szepietowski JC. Tinea capitis in southwest Poland. Mycoses 2008; 52: 193-4.

8. Del Boz-González J. Tinea capitis: trends in Spain. Actas Dermosifiliogr 2012; 103: 288-93.
9. Maraki S, Mavromanolaki VE. Epidemiology of Dermatophytoses in Crete, Greece: a 5 year survey. Med Mycol 2016; 57: 69-75.

10. Silva-Rocha WP, de Azevedo MF, Chaves GM. Epidemiology and fungal species distribution of superficial mycoses in Northeast Brazil. J Mycol Med 2017; 27: 57-64.

11. Baran E, Szepietowski J, Walów E, et al. Fungal skin infections in Lower Silesia in the years 1974-1991 (Zakażenia grzybicze skóry na Dolnym Śląsku w latach 1974-1991). Przegl Dermatol 1993; 80: 49-58.

12. Alshawa K, Lacroix C, Benderdouche M, et al. Increasing incidence of Trichophyton tonsurans in Paris, France: a 15-year retrospective study. BJD 2012; 166: 1121-54.

13. Borman AM, Campbell CK, Fraser M, et al. Analysis of the dermatophyte species isolated in the British Isles between 1980 and 2005 and review of worldwide dermatophyte trends over the last three decades. Med Mycol 2007; 45: 131-41.

14. Leeming JG, Elliot TS. The emergence of Trichophyton tonsurans tinea capitis in Birmingham. Br J Dermatol 1995; 133 : 929-31.

15. Del Boz J, Crespo V, Rivas-Ruiz F, de Troya M. A 30-year survey of paediatric tinea capitis in southern Spain. J Eur Acad Dermatol Venereol 2011; 25: 170-4.

16. Mapelli ET, Cerri A, Bombonato C, Menni S. Tinea capitis in the paediatric population in Milan, Italy: the emergence of Trichophyton violaceum. Mycopathologia 2013; 176: 243-6.

17. Kieliger S, Glatz M, Cozzio A, Bosshard PP. Tinea capitis and tinea faciei in the Zurich area - an 8-year survey of trends in the epidemiology and treatment patterns. J Eur Acad Dermatol Venereol 2014; 29: 1524-9.

18. Paskevicius A, Svediene J. Distribution and species composition of causative agents of dermatophytoses in Lithuania. Acta Dermatovenerol Croat 2013; 21: 99-104.

19. Zamani S, Sadeghi G, Yazdinia F, et al. Epidemiological trends of dermatophytosis in Tehran, Iran: a five-year retrospective study. JMM 2016; 26: 351-8. 